\title{
SOCS1 gene therapy has antitumor effects in imatinib-resistant gastrointestinal stromal tumor cells through FAK/PI3 K signaling
}

\author{
Takahito Sugase $^{1,2,5} \cdot$ Tsuyoshi Takahashi $^{1} \cdot$ Satoshi Serada ${ }^{2} \cdot$ Minoru Fujimoto $^{2} \cdot$ Tomoharu Ohkawara $^{2}$. \\ Kosuke Hiramatsu ${ }^{2} \cdot$ Toshirou Nishida $^{3} \cdot$ Seiichi Hirota ${ }^{4} \cdot$ Yurina Saito $^{1} \cdot \mathrm{Koji}$ Tanaka $^{1} \cdot$ Yasuhiro Miyazaki $^{1}$. \\ Tomoki Makino $^{1} \cdot$ Yukinori Kurokawa $^{1} \cdot$ Makoto Yamasaki $^{1} \cdot$ Kiyokazu Nakajima $^{1} \cdot$ Kazuhiro Hanasaki $^{5}$. \\ Tadamitsu Kishimoto ${ }^{6} \cdot$ Masaki Mori $^{1} \cdot$ Yuichiro Doki ${ }^{1} \cdot$ Tetsuji Naka $^{2}$
}

Received: 27 December 2017 / Accepted: 28 March 2018 / Published online: 5 April 2018

(c) The International Gastric Cancer Association and The Japanese Gastric Cancer Association 2018

\begin{abstract}
Background Most of the gastrointestinal stromal tumors (GIST) have mutations in the KIT gene, encoding a receptor tyrosine kinase. Imatinib, a receptor tyrosine kinase inhibitor, is the first-line therapy for unresectable and metastatic GISTs. Despite the revolutionary effects of imatinib, some patients are primarily resistant to imatinib and many become resistant because of acquisition of secondary mutations in KIT. This study investigated the antitumor effects of SOCS1 gene therapy, which targets several signaling pathways.

Methods We used GIST-T1 (imatinib-sensitive) and GIST-R8 (imatinib-resistant) cells. We infected both cell lines with an adenovirus expressing SOCS1 (AdSOCS1) and examined antitumor effect and mechanisms of its agent.

Results The latter harboured with secondary KIT mutation and had imatinib resistance $>1000$-fold higher than the former cells. We demonstrated that AdSOCS1 significantly decreased the proliferation and induced apoptosis in both cell lines. Moreover, SOCS1 overexpression inhibited the phosphorylation of signal transducer and activator of transcription 3 (STAT3), AKT, and focal adhesion kinase (FAK) in both of them. Inhibition of JAK signaling did not affect the proliferation enough. However, inhibition of the FAK signaling with an FAK inhibitor or RNA interference significantly showed inhibitory effect on cell growth and suppressed the phosphorylation of AKT, indicating a cross-talk between the AKT and FAK pathways in both the imatinib-sensitive and imatinib-resistant GIST cells.

Conclusions Our results indicate that the activation of FAK signaling is critical for proliferation of both imatinib-sensitive and -resistant GIST cells and the interference with FAK/AKT pathway might be beneficial for therapeutic target.
\end{abstract}

Keywords Gastrointestinal stromal tumor $\cdot$ SOCS1 $\cdot$ Imatinib resistance $\cdot$ Focal adhesion kinase $\cdot$ Secondary KIT mutation

\section{Introduction}

Electronic supplementary material The online version of this article (https://doi.org/10.1007/s10120-018-0822-1) contains supplementary material, which is available to authorized users.

Tsuyoshi Takahashi

ttakahashi2@gesurg.med.osaka-u.ac.jp

Tetsuji Naka

tnaka@nibiohn.go.jp

1 Department of Gastroenterological Surgery, Osaka University Graduate School of Medicine, 2-2 Yamadaoka, Suita 565-0871, Osaka, Japan

2 Center for Intractable Disease, Kochi University, Kohasu, Okocho, Nankoku 783-8505, Japan
Gastrointestinal stromal tumor (GIST) is the most common mesenchymal tumor in the gastrointestinal tract $[1,2]$. Most GISTs are characterized by mutations in

3 National Cancer Center Hospital, Tokyo, Japan

4 Department of Surgical Pathology, Hyogo College of Medicine, Nishinomiya, Japan

5 Department of Surgery, Kochi University, Nankoku, Japan

6 Laboratory of Immune Regulation, Osaka University Graduate School of Frontier Biosciences, Suita, Japan 
the proto-oncogene $K I T$, encoding the receptor proteintyrosine kinase KIT that is a key factor in sporadic GIST pathogenesis and proliferation [3,4]. Activated KIT in turn activates various downstream pathways, including the phosphoinositide 3-kinase (PI3 K)/AKT, mitogenactivated protein kinase (MAPK), and Janus kinase (JAK)/ signal transducer and activator of transcription (STAT) pathways, and induces cell proliferation. The PI3 K/ AKT pathway is considered to be more important than the MAPK or JAK/STAT pathways in conducting the KIT oncogenic signal in GISTs [5]. This knowledge has facilitated the design of targeted therapies employing tyrosine kinase inhibitors (TKIs) and, specifically, the development of imatinib mesylate (Glivec ${ }^{\circledR}$, Novartis Pharmaceuticals) for the treatment of GITS. Imatinib mesylate (commonly indicated as imatinib) is a selective inhibitor of KIT, platelet-derived growth factor receptor A (PDGFRA), and Abelson proto-oncogene (ABL) via competitive binding to their adenosine tri-phosphate (ATP)-binding domains: consequently, this drug exerts its antitumor activity by shutting down KIT-mediated signaling. The prognosis for patients with GIST has dramatically improved, since imatinib therapy was introduced to clinical practice [5, 6]. Despite the effectiveness of imatinib, however, several problems remain, such as susceptibility and resistance to imatinib. Although various attempts to treat patients with imatinib-resistant GIST have been made, the success of such treatments is still limited [7]. Most imatinib-resistant GISTs acquire, indeed, secondary KIT mutations and are characterized by re-activation of KIT downstream pathways. Therefore, new approaches that allow the suppression of KIT downstream pathways in imatinib-resistant GIST are needed.

The family of suppressor of cytokine signaling (SOCS), characterized by a central src homology 2 (SH2) domain and a conserved C-terminal SOCS box, is composed of eight structurally related proteins [8]. Of these, SOCS 1 is the most potent negative regulator of pro-inflammatory cytokine signaling and interacts with phosphotyrosine residues on proteins [9]. We have previously reported that overexpression of SOCS1 in cells, using adenoviral vectors, has a potent antiproliferative effect obtained through the targeting of several signaling pathways, including the JAK/STAT, MAPK, focal adhesion kinase (FAK), and p53 pathways [10-14]. Since SOCS1 targets multiple signaling pathways, it may be a promising therapeutic approach for the treatment of several tumors, by preventing and/or circumventing drug resistance in tumor cells [15].

In this study, we investigated the effect of SOCS1 overexpression on the progression of GIST using imatinib-sensitive and -resistant GIST cell lines. In addition, we examined the mechanisms through which SOCS1 induces antitumor effects in GIST.

\section{Materials and methods}

\section{Cell lines}

We previously established the human GIST cell line GISTT1, which has a 57-nucleotide in-frame deletion in KIT exon 11, corresponding to the deletion valine (V)570- tyrosine (Y)578 in the KIT protein [16]. The cell line identity was confirmed by DNA fingerprinting through short tandem repeat profiling, as previously described [17]. The GIST-R8 cell line was established from GIST-T1 cells as an imatinibresistant clone that arose from continuous culturing in $5 \mathrm{nM}$ imatinib. All cell lines were maintained in Dulbecco's Modified Eagle Medium (DMEM) supplemented with $10 \%$ foetal bovine serum (FBS; HyClone Laboratories, Logan, UT, USA), $100 \mathrm{U} / \mathrm{mL}$ penicillin and $100 \mu \mathrm{g} / \mathrm{ml}$ streptomycin (PS; Nacalai Tesque, Kyoto, Japan) at $37^{\circ} \mathrm{C}$, under a humidified atmosphere of $5 \% \mathrm{CO}_{2}$.

\section{Cell proliferation assay}

GIST cells were plated in 96-well plates at a density of $2 \times 10^{3}$ cells per well and incubated for $24 \mathrm{~h}$. Cell proliferation was evaluated using the Cell Counting Reagent SF (Nacalai Tesque) at the indicated times, as previously described $[14,15]$. The following inhibitors were used in this study: Imatinib (Novartis Pharmaceuticals, Basel, Switzerland), JAK inhibitor I (Calbiochem), FAK inhibitor (TAE226, Novartis Pharmaceuticals), and PI3 K inhibitor (LY294002, Cell Signaling Technology, Danvers, MA, USA). TAE226 is a small-molecule inhibitor that was recently synthesized to specifically target FAK: it exerts a potent anti-proliferative effect by suppressing FAK and its downstream effectors [18]. The half maximal inhibitory concentration $\left(\mathrm{IC}_{50}\right)$ values were measured $72 \mathrm{~h}$ after treatment with imatinib and calculated as: $\mathrm{IC}_{50}=10\{\log (\mathrm{A} / \mathrm{B})$ $(50-\mathrm{C}) /(\mathrm{D}-\mathrm{C})+\log (\mathrm{B})\}$ where A represents the higher concentration between the $50 \%$ of the inhibition rate, $\mathrm{B}$ represents the lower concentrations between the $50 \%$ of inhibition rate, $\mathrm{C}$ represents the inhibition rate at concentration $\mathrm{B}$, and $\mathrm{D}$ represents the inhibition rate at concentration $\mathrm{A}$.

A replication-defective recombinant adenoviral vector expressing the human SOCS1 gene (AdSOCS1) was produced by Takara Bio (Kusatsu, Japan). An adenoviral vector expressing the LacZ gene (AdLacZ) was constructed. After incubation in culture medium containing $10 \% \mathrm{FBS}$ and 100 $\mathrm{U} / \mathrm{mL}$ penicillin and $100 \mu \mathrm{g} / \mathrm{mL}$ streptomycin for $24 \mathrm{~h}$, the GIST cells were infected with the indicated adenoviral vectors at a multiplicity of infection (MOI) of 10-160. 


\section{Western blotting analysis}

GIST cell lines were harvested and lysed as previously described, $[14,15]$ and proteins were separated according to standard procedures. The following antibodies were used: anti-phospho (p)-KIT (Tyr703), anti-p-AKT (Thr308), anti-p-AKT (Ser473), anti-AKT, anti-p-p44/42 MAPK, anti-p44/42 MAPK, anti-p-STAT3, and antiCleaved-Caspase3 (1:1000 dilution) were from Cell Signaling Technology (Danvers, MA, USA); anti-KIT and anti-STAT3 (1:1000 dilution), and anti-glyceraldehyde 3-phosphate dehydrogenase (GAPDH, 1:2000 dilution) were from Santa Cruz Biotechnology (Santa Cruz, CA, USA); anti-SOCS1 (1:2000 dilution) was from IBL (Fujioka, Japan); anti-p-FAK (Tyr397) and anti-FAK (1:1000 dilution) were from BD Transduction Laboratories (San Jose, CA, USA).

\section{Caspase-3/7 activity assay}

GIST cells were plated into 96-well white plates at a density of $2 \times 10^{3}$ cells per well and infected with AdLacZ or AdSOCS1 $(\mathrm{MOI}=10$ ) for $48 \mathrm{~h}$. The activity of caspase-3 and -7 in cell culture was detected using the Caspase $\mathrm{Glo}^{\circledR}$ 3/7 Assay kit (Promega, Madison, WI, USA) according to the manufacturer's instructions. Luminometer readings were determined using a Spectra Max Gemini EM Microplate Reader (Molecular Devices, Sunnyvale, CA, USA).

\section{Small-interfering RNA (siRNA) transfection}

The following ON-TARGETplus SMARTpool siRNAs were purchased from Thermo Scientific Dharmacon (Lafayette, CO, USA): non-targeting siRNA (D-00181010-20) and human protein-tyrosine kinase (PTK) 2, a member of the FAK subfamily, siRNA (L-003164- 00-0010). GIST cells were seeded in antibiotic-free DMEM medium with 10\% FBS. The next day, the cells were transfected with the siRNAs using Lipofectamine RNAiMAX transfection reagent (Invitrogen, Carlsbad, CA, USA) according to the manufacturer's instructions. For western blotting analysis, cells were seeded into 6-well plates at a density of $2.5 \times 10^{5}$ cells/well and incubated for $48 \mathrm{~h}$.

\section{Statistical analysis}

Data are shown as the mean \pm standard deviation (SD) for the indicated number of experiments. To test for statistically significant differences between two groups, the unpaired Student's $t$ test was used. Two-sided $P$ values less than 0.05 were considered significant. These analyses were carried out using the JMP software, version 13.0 (SAS Institute, Cary, NC, USA).

\section{Results}

\section{Mutation analysis of GIST-T1 and R8 cells and effect of imatinib treatment}

We have previously established an imatinib-resistant GIST cell line (GIST-R8) by exposing GIST-T1 cells to imatinib [19]. Sanger sequencing revealed that GIST-R8 cells harboured a secondary KIT mutation on exon 17 (D820Y) in addition to a deletion in exon 11 (Fig. 1a). This mutation has been reported as secondary mutation which resulted in imatinib resistance [20]. The imatinib $\mathrm{IC}_{50}$ values for GIST$\mathrm{T} 1$ and R8 cells were $12.2 \mathrm{nM}$ and $15501 \mathrm{nM}$, respectively: the imatinib resistance of GIST-R8 cells was more than 1000-fold higher than that of GIST-T1 cells (Fig. 1b). Next, we evaluated the effect of imatinib on GIST cells by western blotting analysis. The expression of p-KIT was suppressed in response to imatinib treatment in GIST-T1 cells only. KIT downstream pathways, such as the PI3 K, MAPK, and JAK/ STAT pathways, were almost unchanged in GIST-R8 cells compared to those in GIST-T1 cells, upon imatinib treatment (Fig. 1c).

\section{Inhibition of proliferation in GIST cells upon SOCS1 overexpression}

We used a replication-defective recombinant adenoviral vector harbouring SOCS1 (AdSOCS1) to overexpress SOCS1 in GIST cells and investigate its effect on cell proliferation; AdLacZ, expressing the $L a c Z$ gene, was used as a negative control. Both GIST-T1 and R8 cells showed a significant effect on growth inhibition in a concentration-dependent manner in the presence of AdSOCS1 (Fig. 2a). Western blotting analysis showed that the expression of p-STAT3 (Tyr705) was clearly inhibited upon overexpression of SOCS 1 in both cell lines (Fig. 2b). Moreover, SOCS1 overexpression decreased the expression of p-AKT (Thr308 and Ser473) in both cell lines (Fig. 2c). In addition, the levels of total and phosphorylated FAK (Tyr397) were downregulated upon SOCS1 overexpression in GIST-T1 and R8 cells (Fig. 2d).

To evaluate if the overexpression of SOCS1 was associated with the induction of apoptosis, we measured the levels of caspase-3/7 activity using a luciferase luminescence assay after the infection of the cells with AdSOCS1 or AdLacZ. The levels of caspase-3/7 activity in AdSOCS1-infected cells were significantly higher than those in AdLacZ-infected cells (Fig. 3a). In addition, overexpression of SOCS1 was 
a

\begin{tabular}{ccc}
\hline \multirow{2}{*}{ Cell line } & \multicolumn{2}{c}{ c-kit gene mutation } \\
\cline { 2 - 3 } & Primary & Secondary \\
\hline GIST - T1 & $\begin{array}{c}\text { Exon11 } \\
(\text { del })\end{array}$ & - \\
& Exon11 & Exon17 \\
GIST - R8 & $($ del $)$ & (D820Y) \\
\hline
\end{tabular}

b

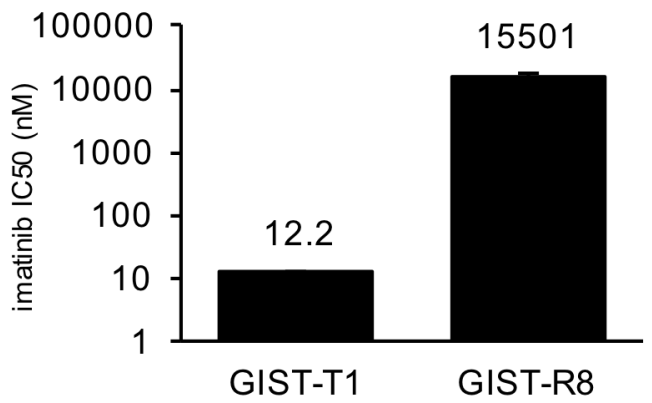

Fig. 1 Mutation analysis in GIST cell lines and effects of imatinib treatment. a KIT gene mutation in two GIST cell lines, GIST-T1 and -R8. b Imatinib $\mathrm{IC}_{50}$ in GIST-T1 and -R8 cells, as determined by

associated with increased expression of cleaved-caspase 3 (Fig. 3b).

\section{JAK inhibition and FAK downregulation suppressed cell proliferation in GIST cells}

To investigate whether the JAK/STAT, FAK, and PI3 K pathways regulated the proliferation of GIST-T1 and R8 cells, we used specific inhibitors of these signaling pathways. Inhibition of the JAK/STAT pathway slightly inhibited the growth of both cell lines, while inhibition of the FAK pathway had a considerable inhibitory effect (Fig. 4a, b). Inhibition of the PI3 K signaling also suppressed cell proliferation in both cell lines (Supplementary Fig. S1). The expression of p-STAT3 (Tyr705) decreased upon treatment of both cell lines with a JAK inhibitor I. High doses of this agent reduced the levels of p-AKT in GIST-T1 cells only and did not change the levels of p-FAK (Fig. 4c). As shown in Fig. 3d, p-FAK levels decreased in both cell lines upon treatment with an FAK inhibitor. In addition, inhibition of the FAK signaling was associated with decreased levels of p-AKT (Thr308 and Ser473) and p-STAT3 and increased levels of cleaved-caspase3 (Fig. 4d).

Next, we examined the association between the FAK signaling and GIST cell proliferation using FAK-directed RNA interference. Attenuation of FAK was associated with the inhibition of cell proliferation in both cell lines, and,
C

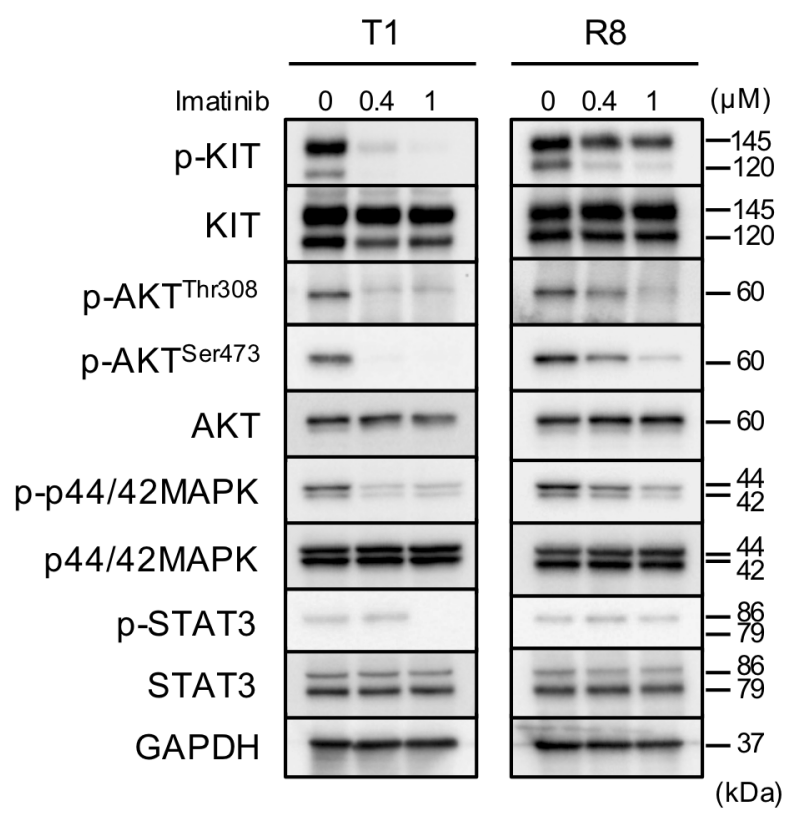

proliferation assays $72 \mathrm{~h}$ after imatinib treatment. c Western blotting analysis $24 \mathrm{~h}$ after imatinib treatment

notably, with a decrease in p-AKT levels even in GIST-R8 cells (Fig. 5a, b).

\section{Discussion}

Malignant GISTs showed that the PI3 K/AKT pathway is the major player in conducting the KIT oncogenic signal in GISTs [5]. We also demonstrated that the inhibition of PI3 K signaling suppresses cell proliferation in imatinib-sensitive and -resistant GIST cells (Supplementary Fig. S1). In addition to these signaling pathways, Koon N. et al. reported that FAK pathway is up-regulated in GISTs and the extent of its upregulation correlates with malignancy and clinical outcome [21]. FAK is a ubiquitously expressed non-receptor protein-tyrosine kinase that has emerged as a crucial molecule involved in the integration of signals from integrins and receptor tyrosine kinases in processes such as cell survival, proliferation, and motility [22]. Recent reports indicated that GIST cells with imatinib resistance had increased FAK signaling and pointed to the inhibition of the FAK pathway as a potential therapeutic strategy for imatinib-resistant GISTs [23, 24].

Regarding the FAK pathway, both the Src homology 2 (SH2) and the kinase inhibitory region (KIR) domains of the SOCS proteins, which are SOCS1 and SOCS3, contribute to FAK binding, and interact with FAK in an FAK-Y397-dependent manner. Moreover, SOCS1 and SOCS3 recruit the 
a
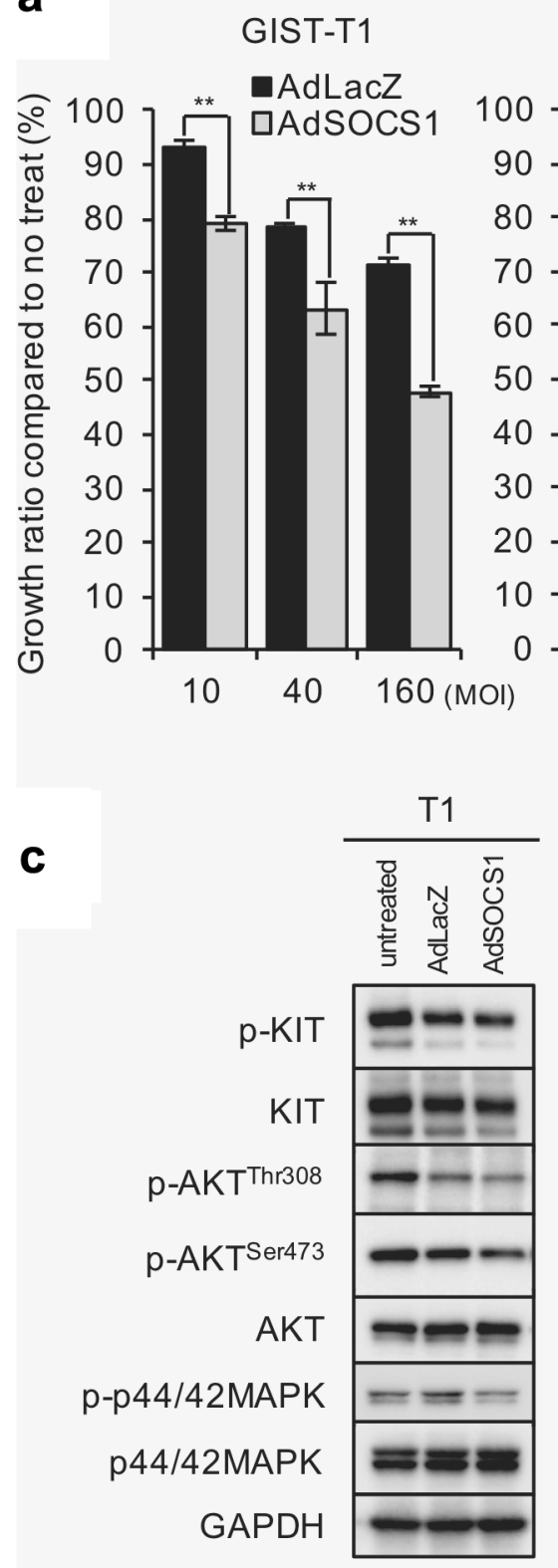

GIST-R8

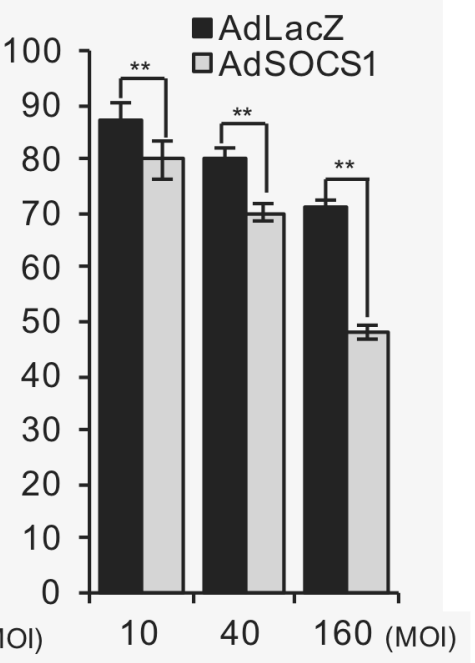

b

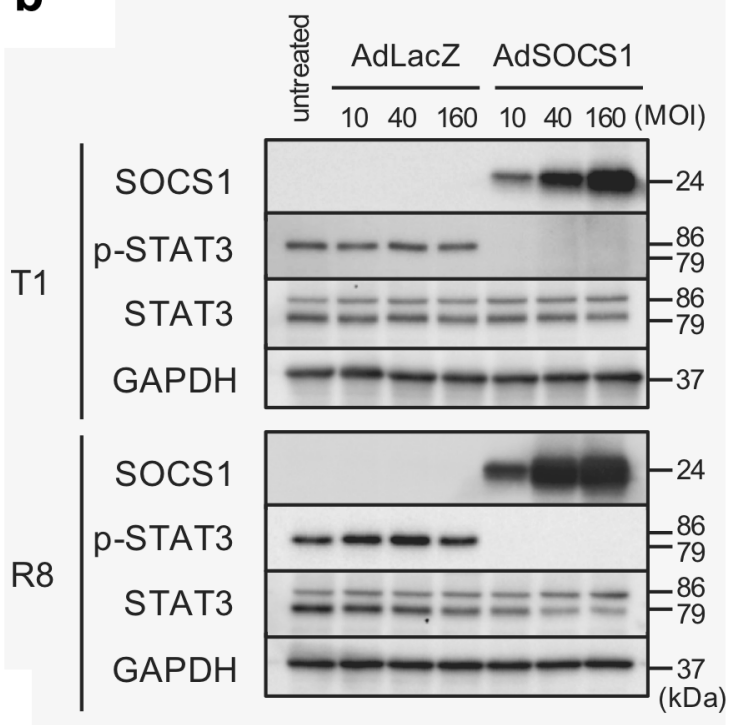

d

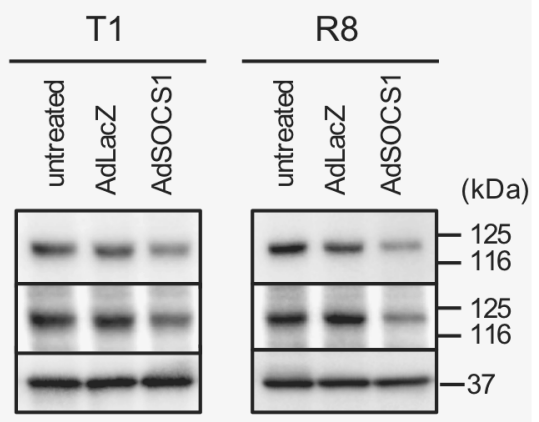

Fig. 2 SOCS1 overexpression inhibits proliferation in GIST cells. a Cell proliferation in GIST-T1 and -R8 cells $72 \mathrm{~h}$ after infection with AdSOCS1 or AdLacZ (negative control) at an MOI of 10-160. b-d
Western blotting analyses showing the expression of the indicated proteins in GIST-T1 and -R8 cells $48 \mathrm{~h}$ after infection with AdSOCS1 or AdLacZ at an MOI of 10-160 (b) or $40(\mathbf{c}, \mathbf{d})$ elongin BC-containing E3 ubiquitin-ligase complex through the conserved SOCS box to promote the degradation of target proteins, such as FAK. SOCS1 promoted polyubiquitination and degradation of FAK in an SOCS box-dependent manner and inhibited FAK-dependent signaling events [25]. In this study, we demonstrated that SOCS1 overexpression was associated with a reduction in the levels of phosphorylated and total FAK and phosphorylated AKT. This effect was clear in both imatinib-sensitive and -resistant GIST cells. Furthermore, inhibition of the FAK pathway through the use of an FAK inhibitor or RNA interference decreased AKT phosphorylation, suggesting a cross-talk between the FAK and AKT/PI3 K pathways. Importantly, our results indicate that, besides the KIT signaling, the activation of the FAK pathway is also critical in both GIST imatinibsensitive and -resistant cells. On the other hand, inhibition of the JAK/STAT pathway did not have anti-proliferative effects enough compared to FAK inhibitor. Therefore, we considered that the clinical effect of STAT 3 inhibitor on GIST patients might not be expected. 
a

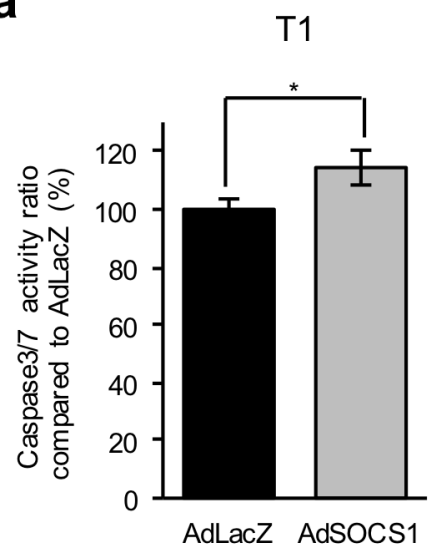

R8

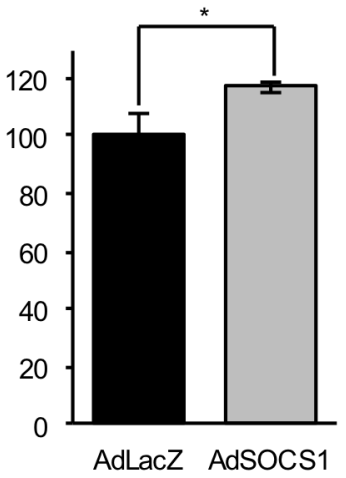

b

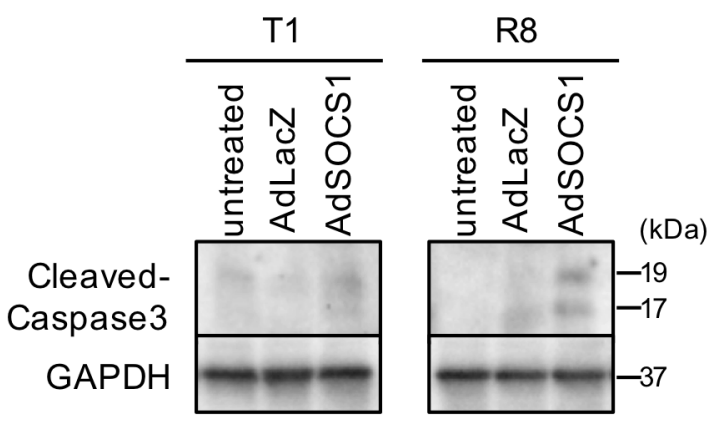

Fig. 3 a Caspase-3/7 activity was determined using luminescence-based assay $48 \mathrm{~h}$ after infection with AdSOCS1 or AdLacZ at an MOI of 10. b Expression of cleaved-caspase $32 \mathrm{~h}$ after infection with AdSOCS1 or AdLacZ at 160MOI

a

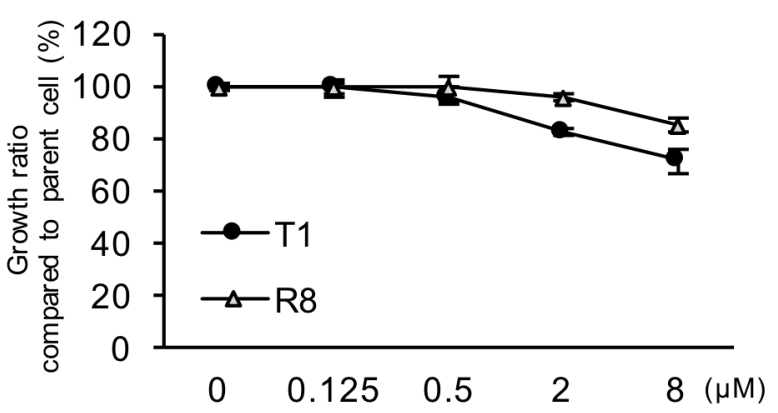

C

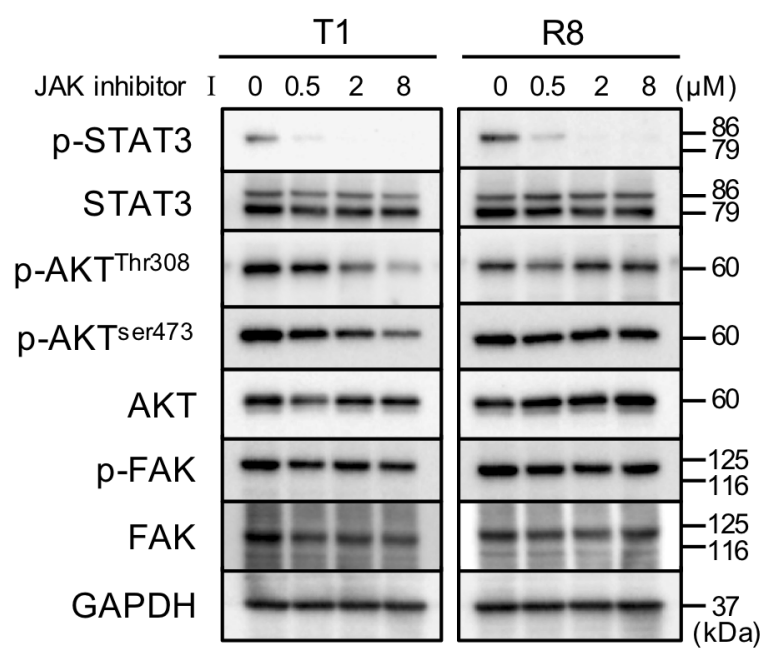

Fig. 4 Cell proliferation was determined $72 \mathrm{~h}$ after treatment of GIST-T1 and -R8 cells with the JAK inhibitor I (a) or a FAK inhibitor $(\mathbf{b})$. Western blotting analyses showing the expression of the indi- b

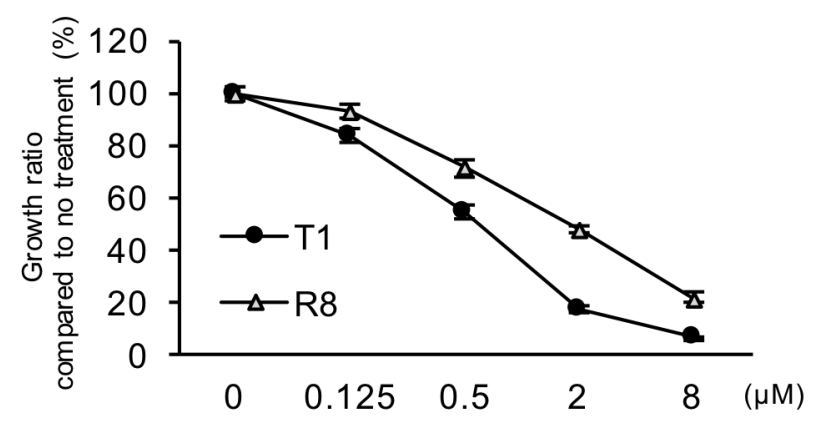

d
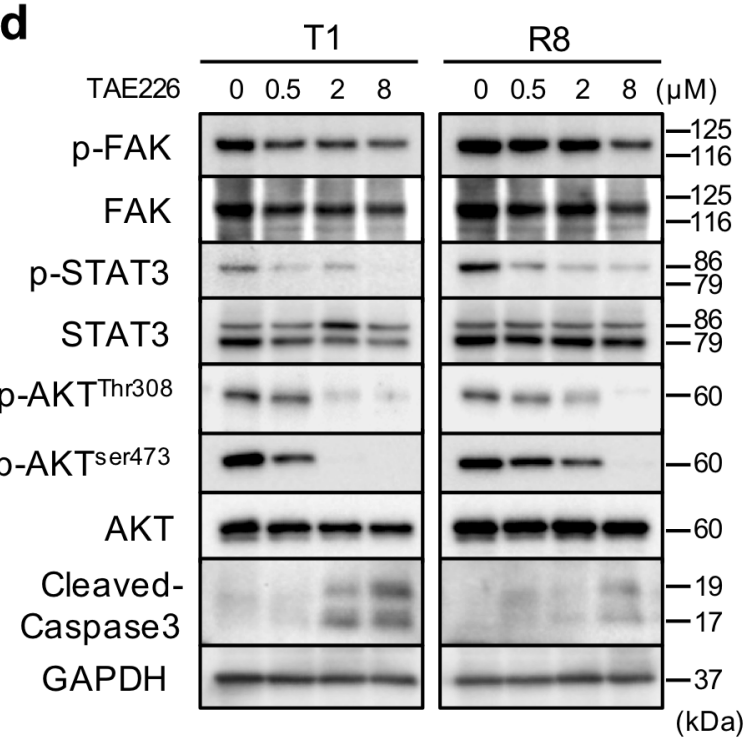

cated proteins in GIST-T1 and -R8 cells $24 \mathrm{~h}$ after treatment of GIST$\mathrm{T} 1$ and -R8 cells with the JAK inhibitor I (c) or a FAK inhibitor (d) 
a

$\mathrm{T} 1$

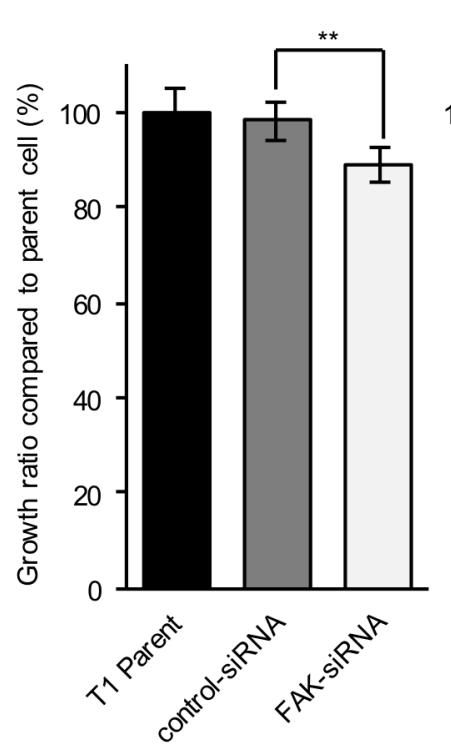

R8

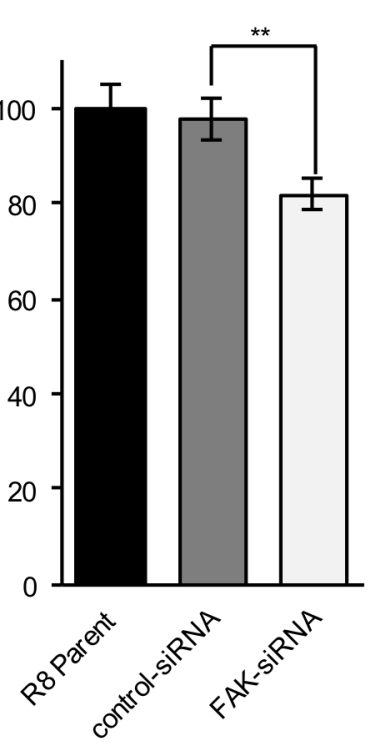

b

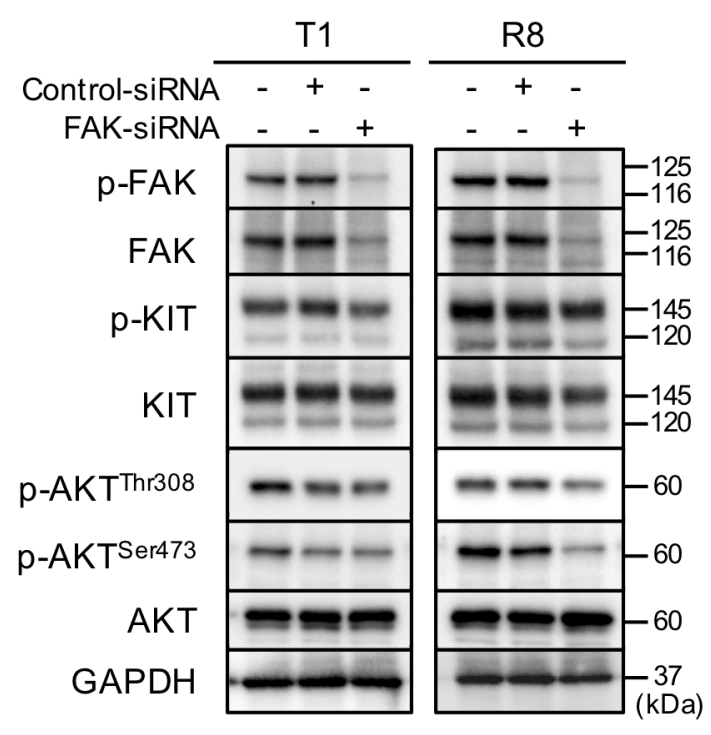

Fig. 5 a Cell proliferation in GIST-T1 and -R8 cells $72 \mathrm{~h}$ after FAK-RNA interference. b Western blotting analysis showing the expression of the indicated proteins in GIST-T1 and -R8 cells $72 \mathrm{~h}$ after FAK-RNA interference

Almost all patients with sporadic advanced or metastatic GIST are treated with imatinib as the first choice $[6,7]$. Although this drug has revolutionized treatment options and significantly improved the clinical outcomes for patients with GIST, there remain several problems. A subset of GIST cases may lose their susceptibility to imatinib, which depends on the specific KIT mutations [26]. Moreover, imatinib-resistant GISTs often show secondary KIT mutations [27]. Approximately $15 \%$ of the patients show primary imatinib resistance, while more than $80 \%$ of the patients have acquired resistance [6]. In this study, we demonstrated that SOCS1 overexpression has antitumor effects other than the inhibition of KIT signaling. Therefore, SOCS1 gene therapy might potentially be beneficial in patients whose KIT signaling is not sufficiently inhibited by treatment with tyrosine kinase inhibitors. Furthermore, patients with GIST cannot discontinue the use of the drug even if complete response is obtained, because it would inevitably lead to re-progression and disease relapse [28]. We have previously reported that SOCS1 strongly induces apoptosis via suppression of anti-apoptotic proteins in various cancers [10-13]. Because SOCS1 overexpression induces apoptosis in both GIST cell lines used in this study, we hypothesize that it might be possible to kill GIST cell that are viable after imatinib therapy by SOCS1 gene therapy. Therefore, SOCS1 gene therapy may represent a new treatment for GISTs, thanks to its antitumor effects. Moreover, the co-treatment with imatinib and SOCS1 gene therapy might improve clinical outcome because of the different antitumor effects of these therapies.
There are some limitations associated with the use of SOCS1 gene therapy in this study. First, we used GISTT1(imatinib-sensitive) and GIST-R8 (imatinib-resistant) which were established from GIST-T1 with continuous imatinib exposure in our laboratory. Although our results using two cell lines might not be enough to confirm definite conclusion, GIST cell lines are generally difficult to establish and making drug-resistant cell lines is even more difficult to establish from drug-sensitive ones. We considered that some data accumulation including animal experiments might be needed to apply clinical trial for SOCS1 gene therapy as a new GIST treatment in the future. Secondary, we used a replication-defective recombinant adenoviral vector, capable to growth and multiply in 293 cells only. Therefore, AdSOCS1 therapy would have its therapeutic effects only in the area of the tumor injected with the virus particles. Although this therapy might be difficult to adapt to GIST patients with multiple distant metastasis such as peritoneal metastasis, imatinib-resistant GIST patients who often showed a single or a small number of resistant lesions, particularly liver metastasis, might benefit from this therapy through endoscopic or percutaneous administration of the viral particles. Moreover, we believe that SOCS1 gene therapy has the advantage that local injection can reduced side effects compared to systemic treatment and perform tumor-specific treatment. Regarding SOCS gene therapy, we are scheduled to begin human clinical trials and the treatment efficacy and safety results are awaited. In addition, we have reported that SOCS1 overexpression has antitumor effect by inhibiting 
various cell growths' signaling including to MAPK [10-15]. Since the FAK inhibition of AdSOCS1 might be only one of the antitumor mechanisms against GIST, we need to further investigate the multiple inhibitory effect of SOCS1 gene therapy in GIST.

In conclusion, SOCS 1 gene therapy, obtained by using an adenoviral vector, suppresses proliferation and induces apoptosis in imatinib-sensitive and -resistant GIST cells. SOCS1 overexpression suppresses the JAK/STAT and FAK/ PI3 K pathways, while it does not affect KIT. In addition, the suppression of the FAK/PI3 K pathway has a significant antitumor effect in imatinib-resistant GIST cells. We consider that more data accumulation is expected to lead to the successful clinical application of SOCS1 gene therapy for GIST treatment.

Acknowledgements This research was partially supported by the Practical Research for Innovative Cancer Control from the Japan Agency for Medical Research and Development (AMED, Grant no. 15ck0106106h0002).

\section{Compliance with ethical standards}

Human rights statement All procedures followed were in accordance with the ethical standards of the responsible committee on human experimentation (institutional and national) and with the Helsinki Declaration of 1964 and later versions.

Animal studies This article does not contain any studies with human or animal subjects performed by any of the authors.

Conflict of interest No author has conflicts of interest that could potentially influence the described research.

\section{References}

1. Joensuu H, Fletcher C, Dimitrijevic S, Silberman S, Roberts P, Demetri G. Management of malignant gastrointestinal stromal tumours. Lancet Oncol. 2002;3:655-64.

2. Colvin H, Mizushima T, Eguchi H, Takiguchi S, Doki Y, Mori M. Gastroenterological surgery in Japan: the past, the present and the future. Ann Gastroenterol Surg. 2017;1:5-10.

3. Hirota S, Isozaki K, Moriyama Y, Hashimoto K, Nishida T, Ishiguro $\mathrm{S}$, et al. Gain-offunction mutations of c-kit in human gastrointestinal stromal tumors. Science. 1998;279:577-80.

4. Nishida T, Hirota S, Taniguchi M, Hashimoto K, Isozaki K, Nakamura $\mathrm{H}$, et al. Familial gastrointestinal stromal tumours with germline mutation of the kit gene. Nat Genet. 1998;19:323-4.

5. Duensing A, Medeiros F, McConarty B, Joseph NE, Panigrahy D, Singer S, et al. Mechanisms of oncogenic KIT signal transduction in primary gastrointestinal stromal tumors (GISTs). Oncogene. 2004;23:3999-4006.

6. Blanke CD, Rankin C, Demetri GD, Ryan CW, von Mehren M, Benjamin RS, et al. Phase III randomized, intergroup trial assessing imatinib mesylate at two dose levels in patients with unresectable or metastatic gastrointestinal stromal tumors expressing the kit receptor tyrosine kinase: S0033. J Clin Oncol. 2008;26:626-32.

7. Debiec-Rychter M, Cools J, Dumez H, Sciot R, Stul M, Mentens $\mathrm{N}$, et al. Mechanisms of resistance to imatinib mesylate in gastrointestinal stromal tumors and activity of the PKC412 inhibitor against imatinib-resistant mutants. Gastroenterology. 2005;128:270-9.

8. Piessevaux J, Lavens D, Peelman F, Tavermier J. The many faces of the SOCS box. Cytokine Growth Factor Rev. 2008;19:371-81.

9. Naka T, Narazaki M, Hirata M, Matsumoto T, Minamoto S, Aono A, et al. Structure and function of a new STAT-induced STAT inhibitor. Nature. 1997;387:924-9.

10. Souma Y, Nishida T, Serada S, Iwahori K, Takahashi T, Fujimoto $\mathrm{M}$, et al. Antiproliferative effect of SOCS-1 through the suppression of STAT3 and p38 MAPK activation in gastric cancer cells. Int J Cancer. 2012;131:1287-96.

11. Shimada K, Serada S, Fujimoto M, Nomura S, Nakatsuka R, Harada E, et al. Molecular mechanism underlying the antiproliferative effect of suppressor of cytokine signaling-1 in non-smallcell lung cancer cells. Cancer Sci. 2013;104:1483-91.

12. Natatsuka R, Takahashi T, Serada S, Fujimoto M, Ookawara T, Nishida T, et al. Gene therapy with SOCS1 for gastric cancer induces $\mathrm{G} 2 / \mathrm{M}$ arrest and has an antitumour effect on peritoneal carcinomatosis. Br J Cancer. 2015;113:433-42.

13. Tagami-Nagata N, Serada S, Fujimoto M, Tanemura A, Nakatsuka $\mathrm{R}$, Ohkawara T, et al. Suppressor of cytokine signalling-1 induces significant preclinical antitumor effect in malignant melanoma cells. Exp Dermatol. 2015;24:864-71.

14. Sugase T, Takahashi T, Serada S, Nakatsuka R, Fujimoto M, Ohkawara T, et al. Suppressor of cytokine signaling-1 gene therapy induces potent antitumor effect in patient-derived esophageal squamous cell carcinoma xenograft mice. Int J Cancer. 2017;140:2608-21.

15. Sugase T, Takahashi T, Serada S, Fujimoto M, Hiramatsu K, Ohkawara T, et al. SOCS1 gene therapy improves radiosensitivity and enhances irradiation-induced DNA damage in esophageal squamous cell carcinoma. Cancer Res. 2017;77:6975-86.

16. Taguchi T, Sonobe H, Toyonaga S, Yamasaki I, Shuin T, Takano A, et al. Conventional and molecular cytogenetic characterization of a new human cell line, GIST-T1, established from gastrointestinal stromal tumor. Lab Invest. 2002;82:663-5.

17. Yokoyama T, Enomoto T, Serada S, Morimoto A, Matsuzaki $\mathrm{S}$, et al. Plasma membrane proteomics identifies bone marrow stromal antigen 2 as a potential therapeutic target in endometrial cancer. Int J Cancer. 2013;132:472-84.

18. Halder J, Lin YG, Merritt WM, Spannuth WA, Nick AM, Honda $\mathrm{T}$, et al. Therapeutic efficacy of a novel focal adhesion kinase inhibitor TAE226 in ovarian carcinoma. Cancer Res. 2007;67:10976-83.

19. Takahashi T, Elzawahry A, Mimaki S, Furukawa E, Nakatsuka $\mathrm{R}$, Nakamura $\mathrm{H}$, et al. Genomic and transcriptomic analysis of imatinib resistance in gastrointestinal stromal tumors. Genes Chromosomes Cancer. 2017;56:303-13.

20. Wardelmann E, Thomas N, Merkelbach-Bruse S, Pauls K, Speidel $\mathrm{N}$, Büttner R, et al. Acquired resistance to imatinib in gastrointestinal stromal tumours caused by multiple KIT mutations. Lancet Oncol. 2005;6:249-51.

21. Koon N, Schneider-Stock R, Sarlomo-Rikala M, Lasota J, Smolkin $\mathrm{M}$, Petroni $\mathrm{G}$, et al. Molecular targets for tumour progression in gastrointestinal stromal tumours. Gut. 2004;53:235-40.

22. Schmidmaier R, Baumann P. ANTI-ADHESION evolves to a promising therapeutic concept in oncology. Curr Med Chem. 2008;15:978-90.

23. Sakurama K, Noma K, Takaoka M, Tomono Y, Watanabe N, Hatakeyama $\mathrm{S}$, et al. Inhibition of focal adhesion kinase as a potential therapeutic strategy for imatinib-resistant gastrointestinal stromal tumor. Mol Cancer Ther. 2009;8:127-34.

24. Takahashi T, Serada S, Ako M, Fujimoto M, Miyazaki Y, Nakatsuka R, et al. New findings of kinase switching in gastrointestinal 
stromal tumor under imatinib using phosphoproteomic analysis. Int J Cancer. 2013;133:2737-43.

25. Liu E, Cote JF, Vuori K. Negative regulation of FAK signaling by SOCS proteins. EMBO J. 2003;22:5036-46.

26. Heinrich MC, Corless CL, Blanke CD, Demetri GD, Joensuu H, Roberts PJ, et al. Molecular correlates of imatinib resistance in gastrointestinal stromal tumors. J Clin Oncol. 2006;29:4764-74.

27. Nishida T, Kanda T, Nishitani A, Takahashi T, Nakajima K, Ishikawa $\mathrm{T}$, et al. Secondary mutations in the kinase domain of the
KIT gene are predominant in imatinib-resistant gastrointestinal stromal tumor. Cancer Sci. 2008;99:799-804.

28. Le Cesne A, Ray-Coquard I, Bui BN, Adenis A, Rios M, Bertucci F, et al. Discontinuation of imatinib in patients with advanced gastrointestinal stromal tumours after 3 years of treatment: an open-label multicenter randomised phase 3 trial. Lancet Oncol. 2010;11:942-9. 